\title{
Infrastructures et espaces urbains en transition
}

Infrastructures and urban spaces in transition

\section{Anne Hecker}

\section{(2) OpenEdition}

Journals

Édition électronique

URL : http://journals.openedition.org/rge/7303

DOI : $10.4000 /$ rge.7303

ISSN : 2108-6478

\section{Éditeur}

Association des géographes de l'Est

Référence électronique

Anne Hecker, «Infrastructures et espaces urbains en transition », Revue Géographique de l'Est [En ligne], vol. 57 / 3-4 | 2017, mis en ligne le 05 octobre 2018, consulté le 10 décembre 2020. URL : http:// journals.openedition.org/rge/7303 ; DOI : https://doi.org/10.4000/rge.7303

Ce document a été généré automatiquement le 10 décembre 2020.

Tous droits réservés 


\title{
Infrastructures et espaces urbains en transition
}

\author{
Infrastructures and urban spaces in transition
}

\author{
Anne Hecker
}

1 Dans le contexte du renouvellement urbain, souvent fortement contraint par la pression foncière et démographique, une nouvelle étape de la fabrique de la ville a été récemment mise en lumière avec le concept d'«urbanisme transitoire ». Nouvelle utilisation, légale mais temporaire, d'espaces rendus vacants par une période de rupture, ils accueillent ponctuellement des opérations à vocation économique, culturelle ou encore sociale. Cette «fabrique urbaine partagée » (IAU IDF ${ }^{1}$ ) relève de la rencontre de trois réalités: au sein de la ville, des besoins sociaux insatisfaits en matière d'espaces publics, particulièrement à vocation artistique; la survenue d'une rupture soulignant une crise, qu'elle soit urbaine, industrielle ou encore économique, qui transforme des espaces fonctionnels en situation de déshérence; et enfin une rupture temporelle, moment d'indécision de la production urbaine, qui place ces espaces ponctuellement entre parenthèses, le temps qu'un aménagement définitif puisse émerger. Si la notion de "transitoire" est ici souvent utilisée comme un synonyme de «provisoire ", les aménagements et projets réalisés se mettent en place lors d'une période de transition d'un site, en vue d'un projet urbain futur (ibid).

2 L'idée transversale qui réunit les cinq articles rassemblés dans ce numéro relève de cette thématique des transitions qui animent la ville et ses infrastructures, et notamment en lien avec le monde des transports. Si les projets et réalisations dont il est question dans ce numéro thématique de la Revue Géographique de l'Est n'ont pas intégré de phase d'urbanisme transitoire reconnue, ils témoignent néanmoins de la rencontre de ces trois points fondamentaux, marqueurs d'un urbanisme et d'infrastructures en phase de transition. Cette dernière désigne en effet « tant le passage graduel d'une situation à une autre, que chacun des degrés intermédiaires qui le scandent " $\left(\right.$ Bavoux $\left.^{2}\right)$, laissant ainsi des traces et conséquences spatiales dont il sera également question dans les articles ici réunis. 


\section{Des transitions multiples}

3 "Passage progressif d'un état à un autre, dans l'espace comme dans le temps» (Ibid), la transition est multiple, et les articles qui forment la trame de ce numéro y posent des regards diversifiés. Trois auteurs mettent ainsi en avant une transition fonctionnelle, symbole de cette rupture conduisant des espaces doués d'une vocation économique à un renouveau et à de nouvelles fonctions, après une phase plus ou moins longue d'attente et de réflexion. On est alors face à une recréation urbaine, intervenant après un profond bouleversement. Denis et Anne Mathis exposent ainsi la mutation d'une ville-citadelle vers une ville militaire, puis vers une ville post-militaire à travers l'exemple de Landau in der Pfalz. Ils soulignent le choc de deux épisodes de démilitarisation, et le processus d'adaptation de l'espace urbain face à ce bouleversement fonctionnel. Il amène la ville à se recréer et à régénérer son tissu urbain, sur fond pourtant d'une crise généralisée de décroissance urbaine. Laurent Guihéry, à travers l'étude de la ville de Leipzig, évoque la transition d'une ville de l'exAllemagne de l'Est vers une économie occidentalisée, et que la modernisation de ses réseaux de transport fait basculer vers des fonctions commerciales renforcées et renouvelées. L'article d'Antoine Beyer met en évidence une double transition fonctionnelle, qui s'alimentent l'une l'autre: il évoque ainsi les ports de l'espace rhénan, appelés à muter sous l'impact de la modernisation notable du transport fluvial et de l'augmentation des tonnages traités. L'espace historiquement dédié aux ports devient insuffisant, leur imbrication dans la trame urbaine qui s'est étendue devient complexe, et le déménagement découle de cette "transition fonctionnelle douce ». D'industrialo-portuaire, la vocation des espaces libérés puis reconquis glisse vers une tertiarisation et une clusterisation comparables à celle des fronts d'eau maritimes. Cette transition accompagne celle des espaces urbains, qui s'accroissent et visent à conquérir ces espaces fonctionnels à des fins urbanistique, au risque de mettre en danger leurs fonctions économiques, pourtant majeures.

4 Cette transition fonctionnelle et urbaine n'est pas sans appeler une transition d'ordre social, portant en elle les prémices de la gentrification, comme l'évoquent Denis et Anne Mathis à Landau, ou Antoine Beyer dans les espaces portuaires des villes rhénanes. Les espaces réaffectés eux-mêmes, mais également les quartiers alentour sont frappés par cette évolution, ainsi que le rappelle Antoine Beyer, lorsque le «cadre urbain s'éloigne du monde du labeur industriel et la manutention portuaire, du bruit et de la poussière ». Cette transition sociale transparaît également dans l'article d'Anne Hecker portant sur la fabrique urbaine de la ville de Nancy au XIXè et début XXème siècles, en lien avec l'apparition des lignes de tramway. Cet article soulève notamment la question d'une potentielle redistribution socio-professionnelle des habitants dans l'espace urbain, rendue possible par les trajets facilités par l'apparition du transport collectif, qui déconstruirait les quartiers ouvriers directement couplés à la localisation de leur employeur.

5 La fabrique de la ville constitue l'inscription spatiale de ces transitions. Ainsi, la pression démographique et migratoire, ainsi que la mutation économique de Nancy y font naître de nouveaux quartiers hors les murs historiques de la ville. Les transports collectifs naissants, à l'origine de mobilités désormais facilitées, vont partiellement contribuer à guider cette urbanisation croissante (Anne Hecker). La transformation des espaces portuaires ou militaires ne se limitent pas quant à eux à une mutation in situ, 
mais donnent là aussi l'occasion à l'espace urbain de se réinventer. Denis et Anne Mathis, Antoine Beyer, Oliver Vergne soulignent l'importance de la transition vers une ville plus "verte " mise en œuvre à cette occasion. La ville "de demain" alimente particulièrement la réflexion de la commune de Landau, où la charte d'Aalborg oriente la recréation urbaine vers davantage de naturalité et une qualité visuelle accrue, dans un cadre général d'écoquartier.

6 L'article d'Olivier Vergne, à travers le projet de Grand Contournement Ouest de Strasbourg, illustre également cette transition idéologique abordée par l'exemple précédent. Du paradigme moderniste des Trente Glorieuses, qui mettait à l'honneur le mode routier et l'aménagement du territoire par les grands axes structurants, l'opinion et le monde politique se sont partiellement tournés vers des enjeux de durabilité et les préoccupations environnementales. En dépit des atouts proposés par le projet sur ces questions, incluant un déclassement de la section urbaine de l'autoroute en boulevard urbain, la requalification des espaces urbanisés alentour, les projets économiques et d'habitat affichant des valeurs "vertes" à engager sur les réserves foncières constituées, la création d'une nouvelle infrastructure routière incarne désormais l'image d'une forme d'aménagement du passé. Là où Leipzig mise sur l'accessibilité et l'amélioration des infrastructures de transport pour redévelopper son tissu urbain, et particulièrement économique (Laurent Guihéry), à Strasbourg, pour les opposants au projet qui tentent de se structurer en ZAD, la fabrique de la ville ne peut plus passer par la réalisation d'une nouvelle infrastructure routière.

\section{Entre rupture et continuum}

7 Le processus transitionnel se nourrit donc de ruptures, temporelles, spatiales, fonctionnelles, idéologiques ou encore urbaines. Elles font écho aux ruptures spatiales que les infrastructures ont elles-mêmes imposées à la ville : les fonctions militaires, industrielles ou de transport ont ainsi imposé leurs discontinuités au tissu urbain, comme le rappellent les articles ici réunis. Mais la rupture se consomme au moment du grand bouleversement, qui amène la ville à se recréer en réponse à un temps difficile la démilitarisation (D. et A. Mathis), la réunification allemande (L. Guihéry), la perte des fonctions industrialo-portuaires (A. Beyer), le traité de Francfort (1871) et l'arrivée des optants (A. Hecker) imposent en retour un temps où la ville se redessine et se reconvertit, tant dans sa forme urbaine que dans ses fonctions.

Mais l'idée même de transition intègre également une dimension temporelle, période plus ou moins longue au cours de laquelle se réalise le passage progressif d'un état à l'autre. On peut dès lors souligner le continuum qui tempère la radicalité de la rupture précédemment évoquée. A. Beyer évoque ainsi la transition fonctionnelle douce qui conduit à « une mutation naturelle et bienvenue des espaces confrontés à l'obsolescence ». De même, la transition vers la ville verte de demain s'entend, à Landau comme à Strasbourg, avec une épaisseur de temps qui favorise la conservation mémorielle des anciennes fonctions et des paysages qui lui sont associés.

9 Sur le plan spatial, le continuum se traduit par l'impérieuse nécessité ressentie par les villes étudiées de "recoudre" les espaces urbains, désunis par les ruptures. La transition entre les quartiers militaires de Landau et le reste de la ville implique l'ouverture de quartiers fermés, assurant "l'intégration du quartier de casernes dans le tissu urbain». A Strasbourg, les projets de requalification d'une autoroute A35 
transformée en boulevard urbain et des terrains qui l'environnent, ramènent ces espaces dans le cadre urbain. De même, dans les villes portuaires de l'espace rhénan, les espaces avoisinants les quartiers reconquis vont être à leur tour concernés par la mutation de leur tissu urbain, souvent à l'origine d'une transition socio-économique. La réponse au besoin de logements, et le dynamisme que ces réalisations urbaines insufflent au marché local de l'emploi, participent également à couturer ces quartiers reconquis au reste de la ville. Quant à Nancy, les projets de croissance imaginés dans les années 1920 ne s'envisagent que convenablement desservis par des infrastructures de transport collectif de qualité, raccrochant des espaces éloignés au tissu urbain constitué.

Enfin, ces étapes de transition laissent dans le paysage les traces « de chacun des degrés intermédiaires qui le scandent ». Ces traces s'avèrent nécessaires au continuum de la fabrique urbaine, et en composent la caution historique. La préservation des marqueurs mémoriels est donc essentielle, ce que les articles réunis mettent en avant. Ainsi, la difficile intégration de réseaux de transport ferroviaire différents, héritages de conditions géopolitiques complexes, et les tentatives avortées de fonçage du tunnel sous la ville restent encore visibles et vivaces à Leipzig, en dépit de la transition vers un réseau moderne et fonctionnel. A Landau, les projets urbains intègrent de larges parts d'un "patrimoine alibi » militaire, et les villes rhénanes conservent les emblèmes des anciennes zones portuaires ("cheminées éteintes, vieilles grues à usage décoratif ou entrepôts transformés en galeries marchandes»). Ces marqueurs favorisent la couture, physique, mais également mémorielle à travers l'épaisseur du temps, des territoires en transition. Autour de ces traces se construit un discours, un récit urbain, constitutif de l'identité de la ville.

11 Le récit urbain peut alors alimenter la projection vers l'avenir et la démarche de transition. Mais la construction en amont d'un projet urbain demeure, à Landau, dans les villes portuaires de l'espace rhénan, ou à Leipzig, un préalable indispensable à la bonne réussite de la démarche de transition. A contrario, les difficultés et oppositions récurrentes au projet de Grand Contournement Ouest de Strasbourg illustrent la délicate mise en œuvre d'un projet ponctuel, souffrant de la division des acteurs, et de l'émergence de stratégies portant moins sur le fond du projet que sur des enjeux géopolitiques locaux. De même, le semi-échec de la première ligne de tramway de Nancy, déconnectée des plans municipaux, évoque cette nécessité d'une vision globale et intégratrice du développement du territoire urbain et de ses infrastructures. Les cinq articles ici réunis s'accordent ainsi tous sur ce point: quelle que soit la qualité des évolutions antérieures et de leur intégration au tissu urbain, la poursuite de la démarche de transition ne peut que s'appuyer sur un projet urbain construit, planifié et global, évitant les démarches opportunistes et ponctuelles. 


\section{NOTES}

1. IAU IDF, 2018, L'urbanisme transitoire, Ile de France 2030, le territoire se transforme, Paris, IAU IDF, Les Carnets pratiques $n^{\circ} 9,88 \mathrm{p}$.

2. BAVOUX J.J., 2014, Dictionnaire d'analyse spatiale, Paris, A. Colin, 607 p. 